\title{
Three-dimensional modeling of frequency- and time-domain electromagnetic methods with induced polarization effects
}

\author{
Youzheng Qi ${ }^{\mathrm{a}, *}$, Hesham El-Kaliouby ${ }^{\mathrm{b}}$, André Revil ${ }^{\mathrm{a}}$, Abdellahi Soueid Ahmed $^{\mathrm{a}}$, \\ Ahmad Ghorbani ${ }^{\mathrm{c}}$, Jianhui $\mathrm{Li}^{\mathrm{d}}$ \\ ${ }^{a}$ Université Grenoble Alpes, Université Savoie Mont Blanc, CNRS, IRD, IFSTTAR, ISTerre, 38000 Grenoble, France \\ ${ }^{\mathrm{b}}$ National Research Centre, Geophysical Sciences Department, Egypt \\ ${ }^{c}$ Yazd University, Department of Mining and Metallurgical Engineering, Yazd, Iran \\ ${ }^{\mathrm{d}}$ China University of Geosciences, Institute of Geophysics and Geomatics, Wuhan, China
}

\section{A R T I C L E I N F O}

\section{Keywords:}

3D electromagnetic modeling

Frequency-domain electromagnetic method (FDEM)

Transient electromagnetic method (TEM)

Induced polarization (IP)

Finite element

\begin{abstract}
A B S T R A C T
Electrical conduction describes the ability of porous media to conduct electrical charges and induced polarization (IP) describes their ability to reversibly store electrical charges. An effective conductivity can be defined as a complex number with frequency-dependent components (i.e., the conductivity is also dispersive). Although IP effects have been observed in frequency- and time-domain electromagnetic (FDEM and TDEM, respectively) data for years, most FDEM and TDEM studies still treat the earth using the conductivity alone (therefore neglecting IP effects). Electromagnetic field data inversion and interpretation require a quantitative three-dimensional modeling with dispersive conductivities, which is still a challenging problem. Using the generic partial-differential-equation solver Comsol Multiphysics's application program interface (API) with Matlab, we have successfully developed three-dimensional FDEM and TDEM modeling with IP effects. Benchmarks are performed using analytical solutions and other numerical techniques. Results with and without IP effects are also compared and analyzed to illustrate the importance of taking IP effects into account. Our modeling could be of great importance in quantitatively studying IP effects in the FDEM and TDEM methods, in developing new field configurations, and also in educational purposes.
\end{abstract}

\section{Introduction}

Induced polarization (IP) phenomenon is caused by the reversible storage of electrical charges associated with the low-frequency $(<10 \mathrm{kHz})$ polarization of the electrical double layer coating the surface of non-metallic grains and by the induced diffuse layer polarization and the intrinsic grain polarization for semi-conductors such as pyrite and magnetite (Schlumberger, 1920; Luo and Zhang, 1998; Revil et al., 2015). The ability of rocks to reversibly store and to release electrical charges has been demonstrated through laboratory- and field-scale experiments, and also through both time- or frequency-domain experiments (e.g., Kemna et al., 2012; Revil et al., 2012). Electromagnetic methods are based on the Maxwell-Faraday induction theory and have been developed into many geophysical methods such as electromagnetic methods in time and frequency domains (TDEM and FDEM, respectively). Since the electromagnetic spectra of FDEM and TDEM methods used in geophysics mostly lie in the spectral band where the IP response dominates the dispersion of the conductivity $(<10 \mathrm{kHz})$, the IP response is also contained in the measured data. In practice IP effects have already been observed in TDEM data (e.g., Weidelt, 1982 among many authors).

Stable, precise, and reliable modeling of electromagnetic responses is very essential to FDEM and TDEM feasibility analysis, survey design, data inversion and interpretation. Easy-flexible modeling can also make the tutorial of FDEM and TDEM methods easier. Recently, it has been strongly advocated that fully quantitative modeling should take IP effects into consideration (El-Kaliouby and Eldiwany, 2004; MacLennan et al., 2013; Marchant et al., 2014; Belliveau and Haber, 2018). However, in the realm of FDEM and TDEM, most investigations are still based on non-polarizable earth where the conductivity is constant along the whole frequency spectra or with time (e.g., Vallée et al., 2011; Auken et al., 2015). Although some researchers have tried to develop TDEM modeling incorporating IP effects, these codes are usually dedicated to specific problem or are not available to the community

\footnotetext{
* Corresponding author.

E-mail addresses: uzhengqi@gmail.com (Y. Qi), hkaliouby@gmail.com (H. El-Kaliouby), andre.revil@univ-smb.fr (A. Revil), abdellahi.soueid-ahmed@univ-smb.fr (A. Soueid Ahmed), aghorbani@yazd.ac.ir (A. Ghorbani), ljhiiicumt@126.com (J. Li).
} 
(Marchant et al., 2014; Cai et al., 2017b). Based on the intrinsic complexity of developing 3D FDEM and TDEM codes and also the difficulty of making the conductivity dispersive during computation, implementing a quantitative 3D FDEM and TDEM modeling with IP effects is still a challenging problem.

Comsol Multiphysics is a generic partial-differential-equation (PDE) solving environment based on the finite element method. In addition to the user-friendly GUI, it also provides a valuable functionality that all the models can be generated into Matlab codes (Comsol, 2017). Endusers can link Matlab with the Comsol server via its application program interface (API) to solve their equations. In recent years, Comsol has been used more and more in the realm of computers and geosciences. Cardiff and Kitanidis (2008) carried out hydraulic inversion using Comsol API with Matlab. Butler and Sinha (2012) showed how Comsol can be used to model gravity, magnetics, DC resistivity and IP methods, and then Butler and Zhang (2016) implemented geophysical electromagnetic methods on Comsol. Li and Smith (2015) also used Comsol to model the radio imaging method with the displacement current.

In this paper, the Comsol AC/DC module is used to carry out the modeling. This module solves the Maxwell's equations under quasistatic approximations and provides quasi-static functionalities such as infinite elements, which makes it very appropriate for geophysical problems (Butler and Sinha, 2012; Butler and Zhang, 2016). We first model the electromagnetic response without IP effects both in frequency and time domains and then we make the earth polarizable to take account of IP effects and check the changes associated with the polarization. In order to validate our approach, we benchmarked our model with both analytical and numerical 3D results. Analyses are finally presented to illustrate the importance of considering IP effects in FDEM and TDEM methods.

\section{Underlying theory}

The functionality of magnetic fields interface in the AC/DC module of Comsol is used for all the simulations in this paper. Both frequencyand time-domain studies can be implemented via this interface. AC/DC module solves the Maxwell's equations by decomposing the magnetic and electric fields into vector and scalar potentials, respectively. In the frequency domain, the governing equations in the quasi-static regime (e.g., Haber et al., 2000; Haber and Ascher, 2001; Ansari and Farquharson, 2014) are

$\nabla \times \nabla \times \mathbf{A}+i \omega \mu_{0} \sigma \mathbf{A}+\mu_{0} \sigma \nabla \phi=\mu_{0} \mathbf{J}_{s}$,

$i \omega \nabla \cdot(\sigma \mathbf{A})+\nabla \cdot(\sigma \nabla \phi)=\nabla \cdot \mathbf{J}_{s}$,

where $\mathbf{A}$ (in $\mathrm{T} \cdot \mathrm{m}$ ) and $\phi$ (in $\mathrm{V}$ ) are the magnetic vector potential and electric scalar potential, respectively. $i=\sqrt{-1}$ is the pure imaginary number (dimensionless), $\omega$ is the angular frequency (in $\mathrm{rad} / \mathrm{s}$ ), $\mu_{0}$ is the free-space magnetic permeability (in $\mathrm{H} / \mathrm{m}$ ), $\sigma$ is the conductivity (in $\mathrm{S}$ / $\mathrm{m}$, treated as a real number when polarization is neglected) and $\mathbf{J}_{s}$ is the external current density (in $\mathrm{A} / \mathrm{m}^{2}$ ). Since magnetic induction $\mathbf{B}$ (in T) or its derivatives $d \mathbf{B} / d t$ (in T/s) is mostly measured for FDEM and TDEM methods, once the vector potential $\mathbf{A}$ has been solved, the magnetic induction can be directly obtained via $\mathbf{B}=\nabla \times \mathbf{A}$.

In the time domain, the governing equations change into the form (e.g., Haber et al., 2004)

$\nabla \times \nabla \times \mathbf{A}+\mu_{0} \sigma \frac{\partial \mathbf{A}}{\partial t}+\mu_{0} \sigma \nabla \phi=\mu_{0} \mathbf{J}_{s}$

$\nabla \cdot\left(\sigma \frac{\partial \mathbf{A}}{\partial t}\right)+\nabla \cdot(\sigma \nabla \phi)=\nabla \cdot \mathbf{J}_{s}$

In order to solve Eqs. (1) and (2) or Eqs. (3) and (4), we consider $\Gamma$ as the outer boundary of the entire domain and $\hat{n}$ the normal vector for $\Gamma$. Natural Dirichlet boundary condition is provided in the AC/DC module by default as $(\hat{n} \times \mathbf{A})_{\Gamma}=0$,

$\phi_{\Gamma}=0$

Note that the magnetic fields interface solves Eqs. (1)-(4) with the default Weyl gauge and a gauge of adding an extra scalar field to impose a divergence constraint to the magnetic vector potential is provided optionally (Comsol, 2017). The default Weyl gauge is used for all the simulations in the paper.

When IP effects are considered, the conductivity becomes a frequency-dependent complex number. In this case Ohm's law can be written as (e.g., Olhoeft, 1985)

$\mathbf{J}=\sigma^{*} \mathbf{E}=\left(\sigma^{\prime}+i \sigma^{\prime \prime}\right) \mathbf{E}$,

where $\mathbf{J}$ denotes the total current density and $\sigma^{*}$ denotes the complex conductivity. The in-phase component $\sigma^{\prime}$ is related to the electromigration of the charge carriers while the quadrature component $\sigma^{\prime \prime}$ denotes their ability to be stored in the material.

To quantitatively describe the spectral IP characteristics of the earth conductivity, the Cole-Cole model is known to prevail over other models by providing a simple yet accurate representation (Cole and Cole, 1941; Tarasov and Titov, 2013). Here we adopt the representation of the Cole-Cole model from Pelton et al. (1978) and the complex conductivity in Eq. (7) can be written as

$\sigma^{*}(\omega)=\sigma_{0}\left[1-M\left(1-\frac{1}{1+(i \omega \tau)^{c}}\right)\right]^{-1}$,

where $0 \leq c \leq 1$ denotes the Cole-Cole exponent (dimensionless), $\tau$ denotes the time constant, $M=\left(\sigma_{\infty}-\sigma_{0}\right) / \sigma_{\infty}$ denotes the intrinsic chargeability (dimensionless) with $0 \leq M \leq 1$, and $\sigma_{0}\left(<\sigma_{\infty}\right)$ and $\sigma_{\infty}$ denote the DC $(\omega \tau<<1)$ and high-frequency $(\omega \tau>>1)$ electrical conductivities, respectively. With the introduced Cole-Cole model, relationships can be found between the Cole-Cole parameters and the medium properties (e.g., Kemna et al., 2012; Revil et al., 2015). ColeCole model was used to generate the complex conductivity of the polarizable half-space or anomalies in our modeling. Note that although the Ohm's law in Eq. (7) has a simple form in the frequency domain, they will change into very complex forms in the time domain, which makes the TDEM modeling with IP effects very challenging as will be shown in the subsequent sections.

\section{FDEM and TDEM modeling without IP effects}

\subsection{Frequency-domain modeling}

In the frequency domain, the source can either be galvanic (grounded electrode pair) or inductive (ungrounded wire loop) and the transmitter waveform is a periodic sine waveform at several different frequencies which usually varies from a fraction of Hertz to several thousands of Hertz. Because high-frequency EM signals attenuate quickly in the subsurface, the transmitting frequencies used in the FDEM method always lie in the range of $0.01-10,000 \mathrm{~Hz}$.

In our simulations, we consider a central-loop configuration where a $50 \mathrm{~m}$ radius loop source located at the interface between the $10^{-6} \mathrm{~S} / \mathrm{m}$ air and a $0.01 \mathrm{~S} / \mathrm{m}$ half-space as an example. The circular loop is constructed directly by the circle geometry under the work plane functionality and the edge current functionality is employed to impose the current into the loop. Because the far-field of any controlled source tends to perform like spherical wave, we built the simulation domain as a sphere and use the work plane functionality to divide it into two hemispheres to represent the air and the ground. There are two ways to enforce the current into the system, i.e., the primary/secondary-field and the total-field method. The former one pre-calculates the primary field of the current source under a simple background such as layered earth and then calculates the secondary field. Although this method has the merit of alleviating the violent field changes near the source, it 

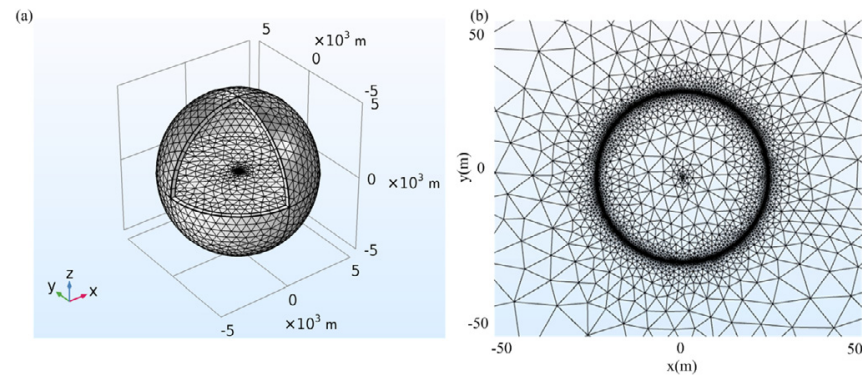

Fig. 1. Geometry of the half-space model. (a) 3D view of the whole domain. (b) Plan view $(z=0 \mathrm{~m})$ of the locally refined mesh around the loop and the central observation point.

cannot take account of rough topographies because no analytical or semi-analytical primary-field expressions can be derived for these complex scenarios (Cai et al., 2017a; Li et al., 2018). Here we choose the total-field method for our modeling that the current is directly enforced into the loop (Ansari and Farquharson, 2014; Cai et al., 2017a,b).

Since wireframes of connected triangles are more accurate and efficient than the conventional rectilinear grid for real-world scenarios (Ansari and Farquharson, 2014; Ansari et al., 2017, Cai et al., 2017a), unstructured free tetrahedral meshes are used to discretize the geometric domain via Delaunay triangulation algorithm. To precisely capture the electromagnetic signal characteristics, locally refined mesh should be used near the source and the observation point. Delaunay refinement method was used to fulfill this job that generates new locally refined meshes each time rather than continuously divide the previous meshes, so the refined size and element quality can be easily controlled. As shown in Fig. 1, the domain near the source and the observation point are locally refined while coarse meshes are used for the remaining parts to make a trade-off between the accuracy and the computational efficiency. Note that because the condition number of the system matrix of electromagnetic modeling strongly depends on the mesh quality, e.g., the minimum dihedral angel and the aspect ratio of the tetrahedral element, extremely elongated meshes should be avoided during the meshing (Ansari and Farquharson, 2014; Cai et al., 2017a). The quality of the meshing in Comsol is mostly determined by the maximum mesh size and the growth rate. Here we set the maximum size of $0.4 \mathrm{~m}$ along the transmitter and receiver and then let the meshes gradually grow to the maximum size of $600 \mathrm{~m}$ to the boundaries of the whole domain. The maximum growth rate of 1.4 is chosen to make the whole elements to be high-quality.

Meanwhile, another challenge in the modeling is how to truncate the simulation domain. If the conventional Dirichlet boundary condition is used, the simulation domain should be sufficiently large that the outer boundary conditions will not affect the solution. Here we resort to the infinite elements in the AC/DC module. Infinite elements accompanied with Dirichlet boundary condition can use mapping functions to stretch the border field quasi-infinitely to alleviate the truncation effect (Comsol, 2017). For one element whose coordinates $\xi$ are normalized from 0 to 1 , the mapping function can be described as

$f(\xi)=\frac{\xi}{\gamma-\xi} \triangle_{p}$

where $\Delta_{p}$ is the diagonal size of the whole geometry and $\gamma$ denotes a number larger than one, computed as

$\gamma=\frac{\Delta_{s}+\Delta_{p}}{\Delta_{s}}$,

where $\Delta_{s}=1000 \Delta_{p}$ is the mapped thickness. Therefore, the coordinate mapping is very nonlinear, progressing from gentle close to the inner border to abrupt toward to quasi-infinite boundary. Since the potentials in the far-field are inversely proportional to the distance from the source, this kind of mapping is suitable for making optimal use of mesh resolution (Zienkiewicz et al., 1983). We used a semi-adaptive method with parametric sweeping of domain size parameter and finally $5 \mathrm{~km}$ sphere with a $300 \mathrm{~m}$ infinite-element layer is chosen for all the simulations. To keep the quality of the infinite elements, each element is meshed into five equally distributed prisms that take the triangles on the inner sphere as the source faces and then sweep radially to the destination.

Once all the physical components are set, a solver will be chosen to solve the finite-element system. This step should be considered with caution because of different performance of the various solvers. Basically the system matrix of the electromagnetic problem is rather illconditioned (Ansari and Farquharson, 2014; Cai et al., 2017a). It is generally accepted that the direct solver, although using more memory, is much more robust than the iterative ones (Cai et al., 2017a; Li et al., 2017, 2018). Our computing environment is $128 \mathrm{~GB}$ RAM, $2.1 \mathrm{GHz}$, and 32 cores. MUMPS direct solver with shared-memory parallelism (OpenMP) is used to accelerate all the simulations in our manuscript. As to the half-space model, we finally generated 165,670 elements with 196,837 unknowns when linear shape functions are chosen as the basis functions. We used $5 \mathrm{~min}$ to get the results of 43 frequencies ranging from 0.01 to $10^{5} \mathrm{~Hz}$ with six frequencies per decade and the physical memory usage was 3 Gbytes. As expected, the employment of infinite elements with Dirichlet boundary condition can greatly reduce the system matrix size and the resulting computational overhead (Puzyrev et al., 2016). The result is compared with the analytical solution provided by Ward and Hohmann (1988) in Fig. 2. Our numerical modeling performs well with less than $2 \%$ relative error.

We continue to validate our modeling by comparison with the benchmark from Newman et al. (1986) that a $2 \mathrm{~S} / \mathrm{m}$ 3D conductive body is embedded in a $0.1 \mathrm{~S} / \mathrm{m}$ half-space. The body is $40 \times 100 \times 30 \mathrm{~m}$ in the $x$-, $y$ - and $z$-directions, respectively, and it is located at a depth of $30 \mathrm{~m}$. The $100 \times 100 \mathrm{~m}$ loop is $50 \mathrm{~m}$ from the position directly over the center of the conductive body and the observation is at the center of the loop (Fig. 3(a)). Since the conductive anomaly itself and the sharp conductivity between the anomaly and the background always causes large field gradients, $10 \mathrm{~m}$ maximum element size and 1.5 maximum growth rate are set in the anomalous region to obtain accurate results. The mesh settings for the transmitter loop and the receiver points are the same as that in the half-space model and finally 178,796 elements with 212,240 unknowns are generated. $11 \mathrm{~min}$ and 3.2 Gbytes memory were used to get the result. As shown in Fig. 3(b), our modeling result coincides well with the benchmark at all the investigated frequencies. As a side note, the result from Newman et al. (1986) was digitized from their article as the original data could not be obtained, so we didn't compute the relative error here because the digitized data are not as accurate as the direct computed ones.

\subsection{Time-domain modeling}

When TDEM method is employed, the transmitter waveform in the
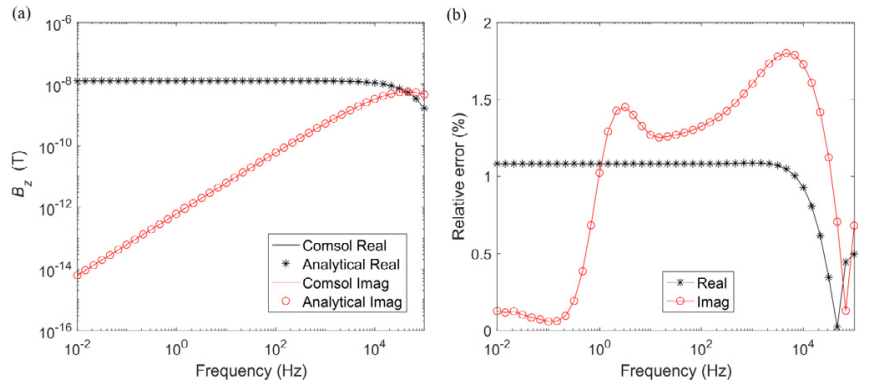

Fig. 2. FDEM responses for the half-space model. The Comsol numerical model and the analytical solution are shown in figure (a). The corresponding relative errors are shown in figure (b). 

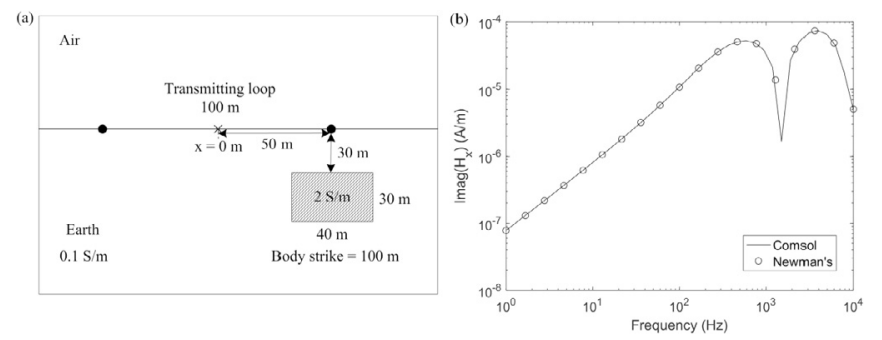

Fig. 3. FDEM responses for a 3D conductive body embedded in a homogenous half-space firstly presented in Newman et al. (1986). (a) A section view of this model. (b) The Comsol numerical model and Newman et al. (1986)'s results are shown for the same geometry at position $x=0 \mathrm{~m}$.

source becomes a time-varying waveform. Since the response of any other waveform can be efficiently calculated by a convolution between the step-off response and the actual transmitter waveform (e.g., Qi et al., 2011; 2017), only step-off current is considered here.

We use the same geometry model and the same mesh as that are used in the frequency domain but with step-off waveform in the circular loop. The difficulty of enforcing the step-off waveform into the system is that there is already a steady constant current before the observation time. We solve this problem by decomposing it into two sub-problems. First, a DC problem is simulated and preserved, and then the established field is used as the initial condition for the following time-dependent simulations. Two studies called stationary study and time-dependent study are automatically carried out in sequence. To get adequate sampling, backward Euler method with the initial step of $10^{-7} \mathrm{~s}$ and strict steps with 100 points per decade from $10^{-6} \mathrm{~s}$ to $0.01 \mathrm{~s}$ is set in the time-dependent solver (Haber et al., 2004). We used $7 \mathrm{~min}$ and 2.2 Gbytes memory to get the solution and compared with the analytical solution from Ward and Hohmann (1988) as shown in Fig. 4. Our modeling gets a good result with the relative error less than $3 \%$, which is usually considered under the noise level in the field observation.

To make our modeling more conclusive, we also compared our modeling with the 3D benchmark from Wang and Hohmann (1993). The model consists of a $3 \mathrm{~S} / \mathrm{m}$ body embedded in a $0.01 \mathrm{~S} / \mathrm{m}$ half-space. The body is $50 \times 100 \times 100 \mathrm{~m}$ and is buried at a depth of $80 \mathrm{~m}$. The $100 \times 100 \mathrm{~m}$ square loop is placed $70 \mathrm{~m}$ from the surface projection over the center of the conductive (Fig. 5(a)). The maximum element size of $15 \mathrm{~m}$ and the maximum element growth rate of 1.45 are set for the anomaly to capture the sharp gradients of the fields in the anomaly and near its boundaries. Finally 247,646 elements with 292,524 unknowns are generated. $11 \mathrm{~min}$ and 2.7 Gbytes memory were used to get the result. The time derivates of the vertical magnetic induction at the center of the loop and $140 \mathrm{~m}$ far from the loop's center are compared with the digitized data from the article of Wang and Hohmann (1993)
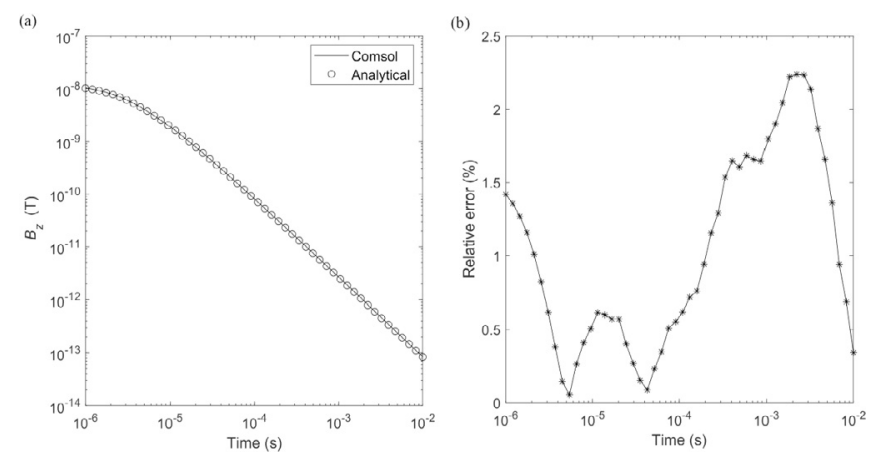

Fig. 4. TDEM responses for the half-space model. The Comsol model and the corresponding analytical solutions are shown in figure (a). The corresponding relative error is shown in Figure (b).
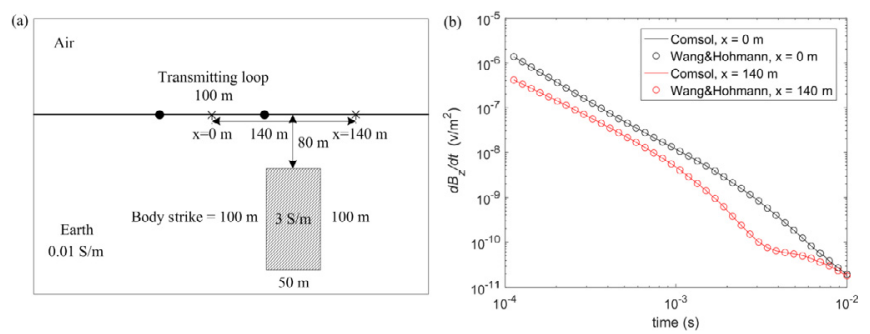

Fig. 5. TDEM responses for a 3D conductive body embedded in a homogenous half-space firstly presented in Wang and Hohmann (1993). (a) A section view of this model. (b) The Comsol numerical model and Wang and Hohmann (1993)'s results are shown for the same geometry at position $x=0 \mathrm{~m}$ and $x=140 \mathrm{~m}$.

as shown in Fig. 5(b). Generally our result agrees well with the benchmark.

\section{FDEM and TDEM modeling with IP effects}

\subsection{Frequency-domain modeling}

When IP effects are considered in the FDEM method, the conductivity becomes frequency-dependent. As for the finite element method used for FDEM modeling with IP effects, such dispersive quality of the conductivity will not change the strong form PDE, the weak form equation and the resulting system matrix compared with that used in the preceding section without IP effects. From the implementation perspective, it is straight-forward to perform FDEM modeling with IP effects in the frequency domain with the only modifications in making the conductivity become frequency-dependent.

We modified the previous half-space model used in the frequencydomain modeling and make the ground become polarizable by a userdefined Cole-Cole model with $M=0.3, c=0.5, \tau=1 \mathrm{~s}, \sigma_{0}=0.01 \mathrm{~S} / \mathrm{m}$ and then compared with the previously computed non-polarizable response with the same conductivity $\sigma=0.01 \mathrm{~S} / \mathrm{m}$. These Cole-Cole parameters lie in the range of typical porous media with metallic particles where obvious IP effects are expected (Revil et al., 2015). Since the same model and mesh were used to model the FDEM with and without IP effects, we found that almost the same time was used to get both results. To show the difference more quantitatively, we can define the relative IP effect as

$\gamma=\left|\frac{f_{\mathrm{IP}}-f_{\text {noIP }}}{f_{\text {noIP }}}\right| \times 100 \%$,

where $f_{\text {IP }}$ and $f_{\text {noIP }}$ are the responses with and without the IP effect, respectively. As shown in Fig. 6 (a), the in-phase response is almost the same as that of the non-polarizable half-space at frequencies less than
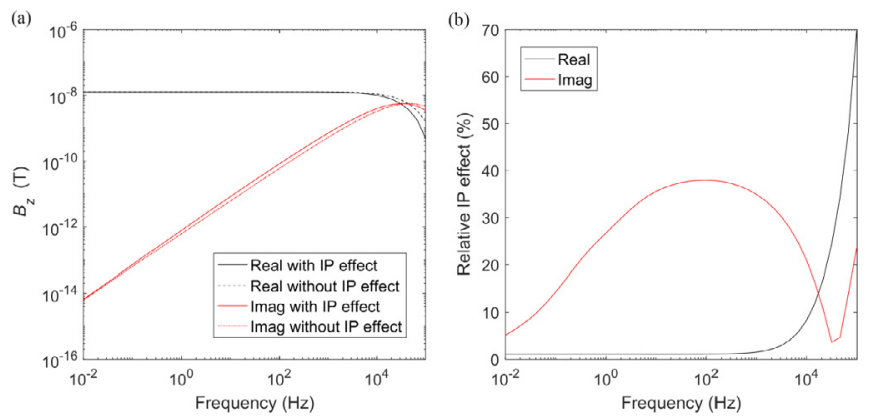

Fig. 6. FDEM responses with and without IP effects for the half-space model. The polarizable half-space are generated by the Cole-Cole model $M=0.3$, $c=0.5, \tau=1 \mathrm{~s}$, and $\sigma_{0}=0.01 \mathrm{~S} / \mathrm{m}$, while the non-polarizable half-space is characterized by the conductivity $\sigma=0.01 \mathrm{~S} / \mathrm{m}$. The responses and the corresponding relative IP effects are shown in figures (a) and (b), respectively. 

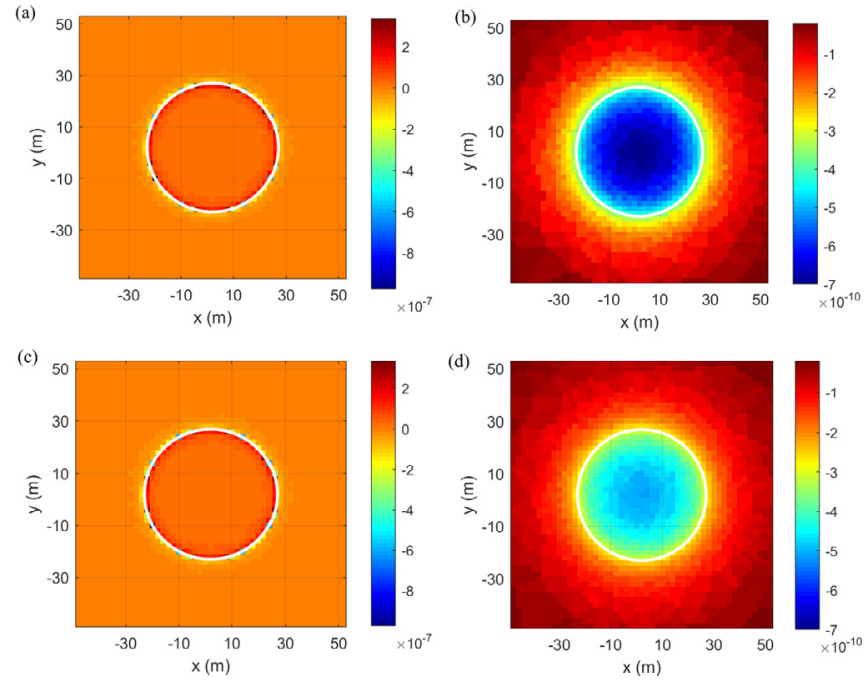

Fig. 7. Vertical component of magnetic induction at the ground surface for the polarizable and non-polarizable half-space at $1000 \mathrm{~Hz}$. (a) and (b) corresponds to the real and imaginary parts of the fields for the polarizable half-space, respectively. (c) and (d) are the real and imaginary parts of the fields for the nonpolarizable half-space. White circles in the figures indicate the loop sources.

$1000 \mathrm{~Hz}(\gamma<5 \%)$ and then goes separately above $1000 \mathrm{~Hz}$, while the quadrature response is greatly distorted by the IP effects, especially between $1 \mathrm{~Hz}$ and $10^{4} \mathrm{~Hz}(\gamma>20 \%)$. To show how IP effects can influence the observed fields on the ground surface, the plan view of the vertical component of the magnetic induction at $1000 \mathrm{~Hz}$ with and without IP effects in the region of $100 \times 100 \mathrm{~m}^{2}$ including the loop source is also shown in Fig. 7. It is noteworthy that the polarizable ground distorts the magnitude of the imaginary part of the measured field very much in this frequency.

In order to highlight the IP effect in the FDEM method, we also studied the response of a 3D polarizable body. We modified the benchmark model from Newman et al. (1986) used in the preceding frequency-domain study. We set the body's previous conductivity $2 \mathrm{~S} / \mathrm{m}$ as the DC conductivity in the Cole-Cole model and also set $M=0.3$, $c=0.5$ and $\tau=1 \mathrm{~s}$ to make the body polarizable. The response is compared with the previous one without IP effects in Fig. 8. It can be seen that the response of the polarizable body differs from that of the conductive body. Note that the second rise after $1 \mathrm{KHz}$ has a different sign from the first rise in each curve. In the first intersection the two lines are of the same sign and in the second of opposite signs, therefore the relative IP effect is near zero for the first intersection and near $200 \%$ for the second. It can be seen that in the low frequency band from $1 \mathrm{~Hz}$
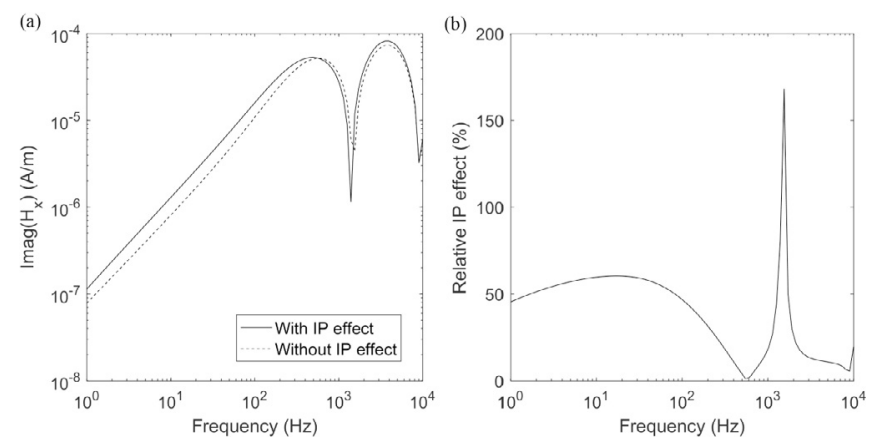

Fig. 8. FDEM responses with and without IP effects for the Newman et al. (1986)'s 3D model. The 3D polarizable body are generated by the Cole-Cole model $M=0.3, c=0.5, \tau=1 \mathrm{~s}$, and $\sigma_{0}=2 \mathrm{~S} / \mathrm{m}$, while the non-polarizable body is still $\sigma=2 \mathrm{~S} / \mathrm{m}$ as in Newman et al. (1986). The responses and the corresponding relative IP effects are shown in (a) and (b), respectively. to $100 \mathrm{~Hz}$ the relative IP effect can be around $50 \%$ which is much bigger than the normal field noise. Neglecting IP effects in such scenarios may cause error to the data inversion and interpretation.

\subsection{Time-domain modeling}

When IP effects are considered in the TDEM method, situation can be much more complex than in the frequency domain. Using inverse Fourier transform to Eq. (7), temporal Ohm's law with IP effects becomes a convolution integral given by

$\mathbf{J}=\int_{-\infty}^{t} \sigma(\tau) \mathbf{E}(t-\tau) d \tau$

Solving such equations directly in the time domain requires the computation of the electrical field at all previous time stages, which makes the problem tedious and impractical (Marchant et al., 2014). There exist two major methods to solve this problem: the direct timedomain and the transformation methods. The former can be implemented by firstly using truncated Padé series to approximate the Cole-Cole model of Eq. (8), then transforming the frequency-domain Ohm's law of Eq. (7) into a set of ordinary differential equations in the time domain by the inverse Fourier transform and finally solving the Maxwell's equations in a time-stepping way (Marchant et al., 2014; Cai et al., 2017b). Recently Belliveau and Haber (2018) firstly introduced the stretched exponential function to describe the IP characteristics and then a direct time-stepping scheme is successfully employed to compute the 3D TDEM response with IP effects. Promising results have also been obtained. However, using the direct time-domain method the internal governing equations of Eqs. (3) and (4) become invalid and thus it cannot be implemented in the AC/DC module. Here we resort to the latter method. We first explicitly solve the response in the frequency domain and then transformed them into the time domain. Although this can be carried through the inverse fast Fourier transform (FFT) method, it has been proved that application of inverse FFT to this problem can require several tens of thousands of kernel evaluations to obtain an accurate response (Newman et al., 1986; Key, 2012). It is common in the electromagnetic geophysical literature to invoke the fast cosine transform (FCT) (Newman et al., 1986; Key, 2012)

$f(t)=-\frac{2}{\pi} \int_{0}^{\infty} \frac{\operatorname{Im}[F(\omega)]}{\omega} \cos (\omega t) d \omega$,

where $\operatorname{Im}[F(\omega)]$ is the imaginary part of the electromagnetic field component in the frequency domain and $f(t)$ the field component in time domain. Eq. (13) can be formed into a linear digital filter and then can be efficiently calculated. In our program 201 filter coefficients from Key (2012) are used to transform the data from the frequency domain to the time domain. The frequency-domain responses are sparsely evaluated at 43 frequencies, distributed from $0.01 \mathrm{~Hz}$ to $10^{5} \mathrm{~Hz}$ with six frequencies per decade.

To validate the FCT method in our modeling, we first transformed the previously computed frequency-domain response without IP effects and compared it with the analytical response from Ward and Hohmann (1988) as shown in Fig. 9. The relative error of the response from FCT method is mostly under the commonly accepted field noise level $3 \%$. In the same fashion, we transformed the previously computed frequencydomain response with IP effects into the time domain. The obtained temporal response with IP effects is shown in Fig. 10. We can observe a sign reversal at the late time. This phenomenon has been discovered in the TDEM field data and is concluded to be caused by the IP effect (Weidelt, 1982). The plan view of the vertical components of the magnetic induction on the ground surface at $10^{-4} \mathrm{~s}$ and $0.01 \mathrm{~s}$ with and without IP effects are compared in Fig. 11. Note that as only the region near the central point is refined the fields are not shown in a smooth way across the whole surface. Here we only want to show the main field features in the vicinity of the loop rather than to measure the accurate field of the whole surface. It is notable that the polarizable earth 

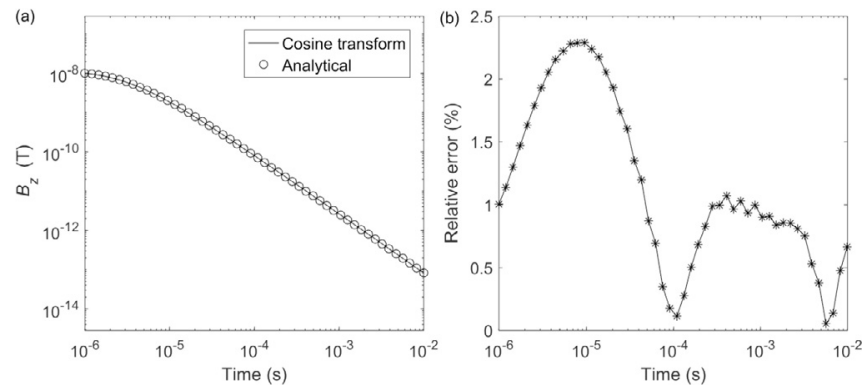

Fig. 9. TDEM responses for the half-space model. The fast cosine transformed and the corresponding analytical solutions are shown in (a). The relative error is shown in (b).
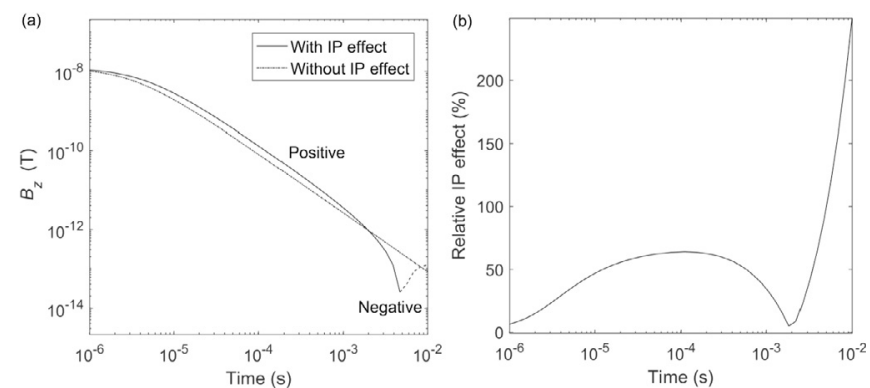

Fig. 10. TDEM responses with and without IP effects for the half-space model. The polarizable half-space is characterized by a Cole-Cole model with $M=0.3$, $c=0.5, \tau=1 \mathrm{~s}$, and $\sigma_{0}=0.01 \mathrm{~S} / \mathrm{m}$, while the non-polarizable half-space is characterized by $\sigma=0.01 \mathrm{~S} / \mathrm{m}$. Responses and the relative IP effects are shown in (a) and (b), respectively.
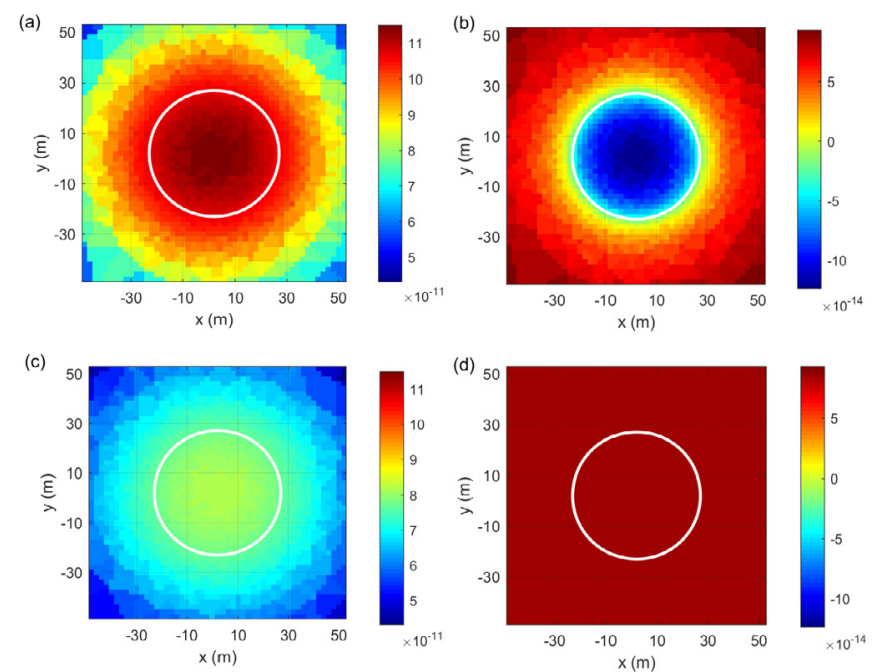

Fig. 11. Vertical component of magnetic induction at the ground surface for the polarizable and non-polarizable half-space at different times. (a) and (b) are the fields for the polarizable half-space at $10^{-4} \mathrm{~s}$ and $0.01 \mathrm{~s}$, respectively. (c) and (d) are the fields for the non-polarizable half-space at $10^{-4} \mathrm{~s}$ and $0.01 \mathrm{~s}$, respectively. The white circles in the figures indicate the loop sources.

distorts the measured field on the ground surface a lot. At the time $0.01 \mathrm{~s}$, opposite to the non-polarizable half-space, the field inside the loop with IP effects demonstrates a different sign.

To show the IP effect in the TDEM method more clearly, we also studied the response of a 3D polarizable body. We modified the benchmark model from Wang and Hohmann (1993) used in the preceding time-domain study by setting $\sigma_{0}=3 \mathrm{~S} / \mathrm{m}, M=0.3, c=0.5$ and $\tau=1 \mathrm{~s}$ to make the body polarizable. The DC conductivity of the polarizable body is the same as the conductivity of the non-polarizable
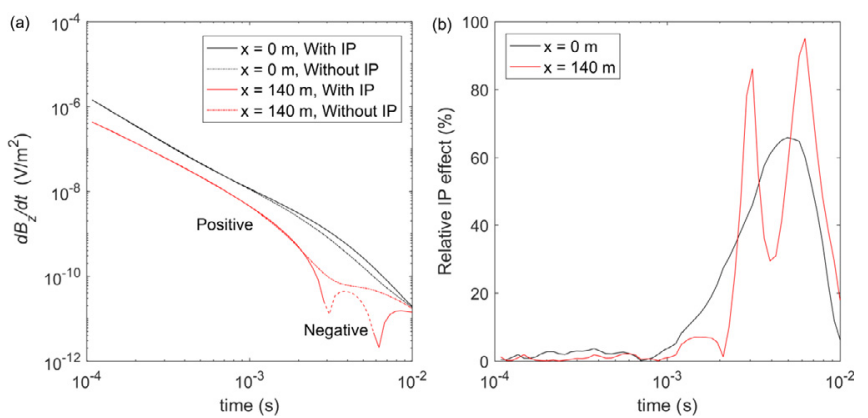

Fig. 12. TDEM responses with and without IP effects for the Wang and Hohmann (1993)'s 3D model. The 3D polarizable body is characterized by the Cole-Cole parameters $M=0.3, c=0.5, \tau=1 \mathrm{~s}$, and $\sigma_{0}=3 \mathrm{~S} / \mathrm{m}$, while the nonpolarizable body still keeps $\sigma=3 \mathrm{~S} / \mathrm{m}$ as in Wang and Hohmann (1993). Responses and the relative IP effects are shown in (a) and (b), respectively.

body. The response is compared with the previous response without IP effects in Fig. 12. It can be seen again that the responses of the polarizable body at the two observation point both differ from that of the non-polarizable body at the late times. Double sign reversals can be clearly seen for the observation point $x=140 \mathrm{~m}$, which clearly shows that the IP effects can play an important role in TDEM data.

Finally, we built a realistic model with complex geometry and topography based on the scaled Grand Bois slag heap field located in the commune of SaintVincent sur L'Isle (France). The surface topography in the vicinity of the $50 \times 50$ square loop source is shown in Fig. 13(a) and the slag anomaly is an approximate $35 \times 40 \times 10 \mathrm{~m}^{3}$ pod under $4 \mathrm{~m}$ of overburden as shown in Fig. 13(b) (Qi et al., 2018). The complex conductivity of the anomaly is considered to be polarizable as $\sigma_{0}=3 \mathrm{~S} / \mathrm{m}, M=0.25, c=0.3$ and $\tau=10 \mathrm{~s}$, and the background are considered to be $0.001 \mathrm{~S} / \mathrm{m}$ based on the isosurface of the time-domain IP data inversion (Qi et al., 2018). Fixed-loop configuration with 64 measuring points inside and outside the loop is studied in this model (Fig. 13(a)). Using the unstructured tetrahedral mesh with locally refined scheme in the transmitter, receivers and anomaly, and providing infinite elements on the border, we finally got 224,658 elements with 265,790 unknowns. Because there were no real FDEM or TDEM survey data for this model, we internally double checked our frequency- and time-domain results via FCT and time-stepping method when the slag anomaly is considered pure conductive with $\sigma=3 \mathrm{~S} / \mathrm{m}$. The FDEM result uses 15 min with 3.5 Gbytes memory while the TDEM results uses 10 min with 3 Gbytes. Three example points are chosen in and out of the loop as shown in Fig. 13(a) and their FCT responses are compared with the direct time-stepping responses as shown in Fig. 14. The superimposition of the three curves with less than $3 \%$ relative errors validate our simulation internally. When the conductivity of the slag anomaly becomes dispersive, the FDEM responses for the three example points can be seen in Figs. 15 and 16, the imaginary parts of the
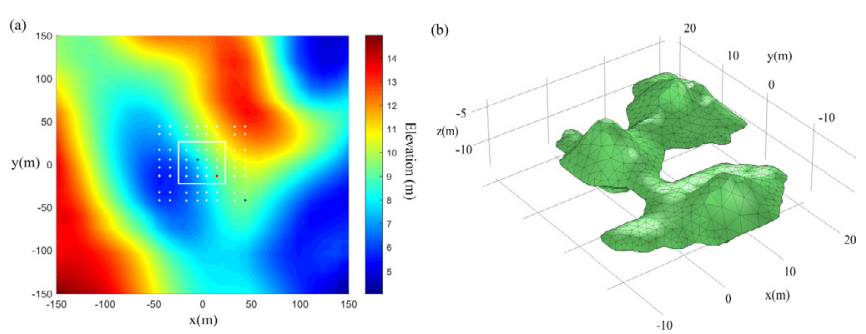

Fig. 13. Grand Bois field located in the commune of SaintVincent sur L'Isle (France). (a) Surface topography of the core region. (b) 3D view of the anomalous slag body. The white square in (a) indicates the transmitting loop with 64 white observation points inside and outside among which the three example points, i.e., point 1 , point 2 and point 3 , to show the response in the paper are filled with black, red and blue, respectively. 

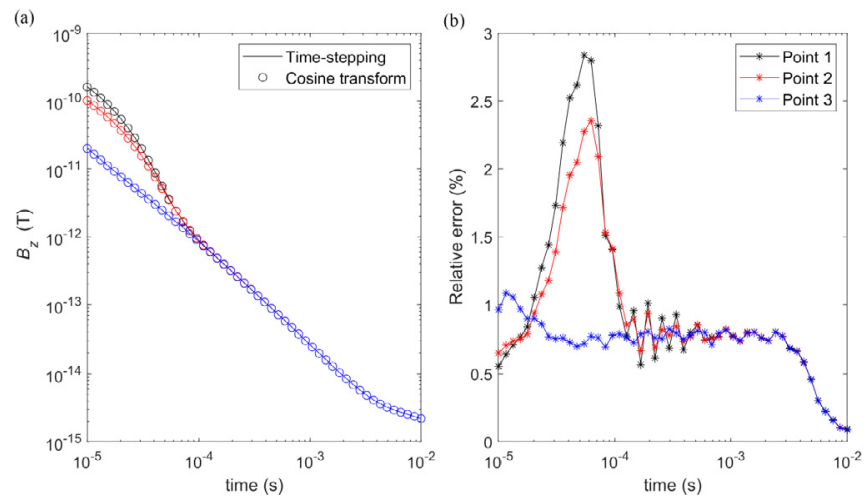

Fig. 14. TDEM responses for the three example points for the Grand Bois model. The time-stepping and fast cosine transformed results are shown in (a). Their relative errors are shown in (b). Note that the color of the response curves here is the same as that is used to indicate the three points amongst the 64 points as shown in Fig. 13.
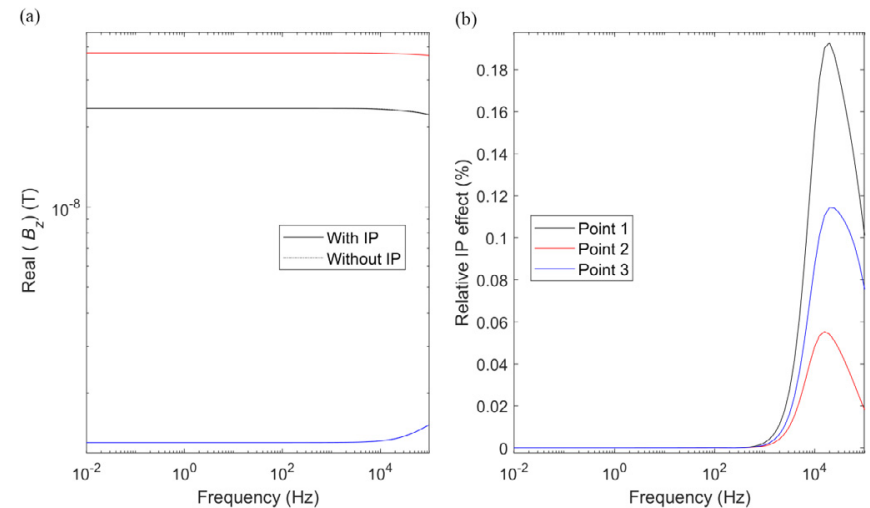

Fig. 15. Real parts of the FDEM responses with and without IP effects for the Grand Bois model. The responses of the three example points and their corresponding relative IP effects are shown in figures (a) and (b), respectively.
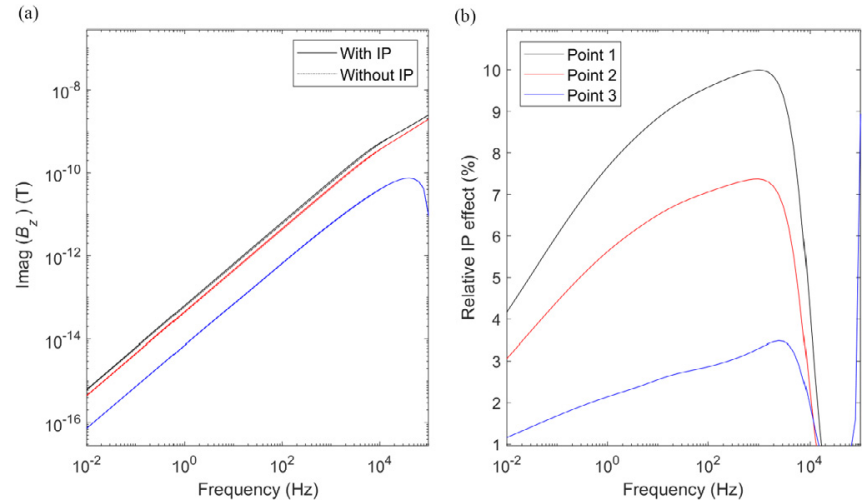

Fig. 16. Imaginary parts of the FDEM responses with and without IP effects for the Grand Bois model. The responses of the three example points and their corresponding relative IP effects are shown in figures (a) and (b), respectively.

responses are affected by the IP effects while the real parts almost show no IP effects for the three points. While in the time domain as shown in Fig. 17, the IP effects are more obviously that the sign changes happen for two out of the three example points which manifest strong relative IP effects with the maximum value that reaches $200 \%$. In this realworld example, we can see again that the IP effects can change the FDEM and TDEM responses to a great extent and they shall be considered in these situations.
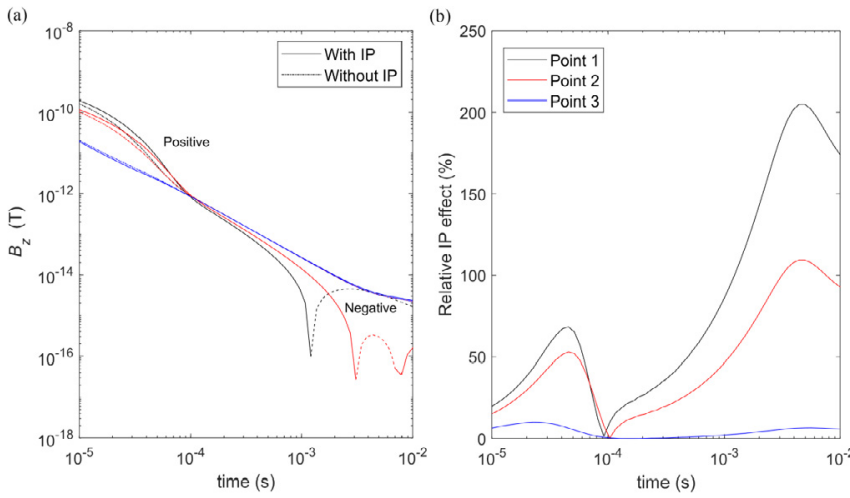

Fig. 17. TDEM responses with and without IP effects for the Grand Bois model. The responses of the three example points and their corresponding relative IP effects are shown in figures (a) and (b), respectively.

\section{Conclusions}

To fully consider IP effects in the FDEM and TDEM methods, we have successfully implemented three-dimensional modeling with IP effects both in frequency and time domains using the generic PDE solver Comsol. For the product form of Ohm's law with IP effects in the frequency domain, we solved the potential Helmholtz equations directly, while for the convolution form of Ohm's law with IP effects in the time domain, we computed the frequency-domain field at sparse frequencies and then transformed them into the time domain via fast Cosine transform. We benchmarked our modeling and also compared with the responses without IP effects. We found that the IP effects can play an important role in both frequency- and time-domain data. Neglecting IP effects may generate spurious inversion results and interpretations. Our modeling can be useful in field configuration design, data inversion, and also for tutorial illustrations of IP effects in FDEM and TDEM methods.

\section{Authorship statement}

Y. Qi conceived the idea, implemented the project and wrote the manuscript. H. El-Kaliouby conceived the idea, discussed the results and improved the manuscript. A. Revil supervised the project, conceived the idea and improved the manuscript. A. Soueid Ahmed, A. Ghorbani and J. Li analyzed and discussed the results.

\section{Acknowledgement}

We thank the Conseil Général de la Dordogne for the financial contribution to this study. We also thank the University of Melbourne through a project funded by the Commonwealth of Australia (contract CR-2016-UNIV.MELBOURNE-147672-UMR5275) and the Egypt-France scientific and technological cooperation program IMHOTEP (project 39464NC). Thanks also go to the editors and the five reviewers. Their comments helped us improve the quality of our manuscript.

\section{References}

Ansari, S., Farquharson, C.G., 2014. 3D finite-element forward modeling of electromagnetic data using vector and scalar potentials and unstructured grids. Geophysics 79, E149-E165.

Ansari, S.M., Farquharson, C.G., MacLachlan, S.P., 2017. A gauged finite-element potential formulation for accurate inductive and galvanic modelling of 3-D electromagnetic problems. Geophys. J. Int. 210, 105-129.

Auken, E., Christiansen, A.V., Kirkegaard, C., Fiandaca, G., Schamper, C., Behroozmand, A.A., Binley, A., Nielsen, E., Effersø, F., Christensen, N.B., Sørensen, K., 2015. An overview of a highly versatile forward and stable inverse algorithm for airborne, ground-based and borehole electromagnetic and electric data. Explor. Geophys. 46, 223-235.

Belliveau, P., Haber, E., 2018. Coupled simulation of electromagnetic induction and 
induced polarization effects using stretched exponential relaxation. Geophysics 83, WB109-WB121.

Butler, S.L., Sinha, G., 2012. Forward modeling of applied geophysics methods using Comsol and comparison with analytical and laboratory analog models. Comput. Geosci. 42, 168-176.

Butler, S.L., Zhang, Z., 2016. Forward modeling of geophysical electromagnetic methods using Comsol. Comput. Geosci. 87, 1-10.

Cai, H., Hu, X., Li, J., Endo, M., Xiong, B., 2017a. Parallelized 3D CSEM modeling using edge-based finite element with total field formulation and unstructured mesh. Comput. Geosci. 99, 125-134.

Cai, H., Hu, X., Xiong, B., Zhdanov, M.S., 2017b. Finite-element time-domain modeling of electromagnetic data in general dispersive medium using adaptive Padé series. Comput. Geosci. 109, 194-205.

Cardiff, M., Kitanidis, P.K., 2008. Efficient solution of nonlinear, underdetermined inverse problems with a generalized PDE model. Comput. Geosci. 34, 1480-1491.

Cole, K.S., Cole, R.H., 1941. Dispersion and absorption in dielectrics. J. Chem. Phys. 9, 341-351.

Comsol Multiphysics User's Guide, 2017. Version 5.3a. Comsol AB, Stockholm, Sweden.

El-Kaliouby, H., Eldiwany, E., 2004. Transient electromagnetic responses of 3D polarizable body. Geophysics 69, 426-430.

Haber, E., Ascher, U.M., 2001. Fast finite volume simulation of 3D electromagnetic problems with highly discontinuous coefficients. SIAM J. Sci. Comput. 22, 1943-1961.

Haber, E., Ascher, U.M., Aruliah, D.A., Oldenburg, D.W., 2000. Fast simulation of 3D electromagnetic problems using potentials. J. Comput. Phys. 163, 150-171.

Haber, E., Ascher, U.M., Oldenburg, D.W., 2004. Inversion of 3D electromagnetic data in frequency and time domain using an inexact all-at-once approach. Geophysics 69 (5), $1216-1228$.

Kemna, A., Binley, A., Cassiani, G., Niederleithinger, E., Revil, A., Slater, L., Williams, K.H., Orozco, A.F., Haegel, F.H., Hoerdt, A., Kruschwitz, S., 2012. An overview of the spectral induced polarization method for near-surface applications. Near Surf. Geophys. 10, 453-468.

Key, K., 2012. Is the fast Hankel transform faster than quadrature? Geophysics 77, F21-F30.

Li, Y., Smith, R.S., 2015. Forward modeling of radio imaging (RIM) data with the Comsol RF module. Comput. Geosci. 85, 60-67.

Li, J., Farquharson, C.G., Hu, X., 2017. 3D vector finite-element electromagnetic forward modeling for large loop sources using a total-field algorithm and unstructured tetrahedral grids. Geophysics 82, E1-E16.

Li, J., Lu, X., Farquharson, C.G., Hu, X., 2018. A finite-element time-domain forward solver for electromagnetic methods with complex-shaped loop sources. Geophysics 83 (3), E117-E132.

Luo, Y., Zhang, G., 1998. Theory and Application of Spectral Induced Polarization. SEG.
MacLennan, K., Karaoulis, M., Revil, A., 2013. Complex conductivity tomography using low-frequency crosswell electromagnetic data. Geophysics 79, E23-E38.

Marchant, D., Haber, E., Oldenburg, D.W., 2014. Three-dimensional modeling of IP effects in time-domain electromagnetic data. Geophysics 79, E303-E314.

Newman, G.A., Hohmann, G.W., Anderson, W.L., 1986. Transient electromagnetic response of a three-dimensional body in a layered earth. Geophysics 51, 1608-1627.

Olhoeft, G.R., 1985. Low-frequency electrical properties. Geophysics 50, 2492-2503.

Pelton, W.H., Ward, S.H., Hallof, P.G., Sill, W.R., Nelson, P.H., 1978. Mineral discrimination and removal of inductive coupling with multifrequency IP. Geophysics 43, 588-609.

Puzyrev, V., Koric, S., Wilkin, S., 2016. Evaluation of parallel direct sparse linear solvers in electromagnetic geophysical problems. Comput. Geosci. 89, 79-87.

Qi, Y., Huang, L., Wu, X., Zhu, W., Fang, G., 2011. The study of arbitrary waveform response in transient electromagnetic method. In: IEEE 4th International Symposium on MAPE, Beijing, 1-3, November 2011, pp. 179-182.

Qi, Y., Huang, L., Wu, X., Zhu, W., Fang, G., Yu, G., 2017. Full waveform modeling of transient electromagnetic response based on temporal interpolation and convolution method. Pure Appl. Geophys. https://doi.org/10.1007/s00024-017-1627-6.

Qi, Y., Soueid Ahmed, A., Revil, A., Ghorbani, A., Abdulsamad, F., Florsch, N., Bonnenfant, J., 2018. Induced polarization response of porous media with metallic particles-Part 7: detection and quantification of buried slag heaps. Geophysics 83, E277-E291.

Revil, A., Karaoulis, M., Johnson, T., Kemna, A., 2012. Some low-frequency electrical methods for subsurface characterization and monitoring in hydrogeology. Hydrogeol. J. 20, 617-658.

Revil, A., Florsch, N., Mao, D., 2015. Induced polarization response of porous media with metallic particles - Part 1: a theory for disseminated semiconductors. Geophysics 80 (5), D525-D538.

Schlumberger, C., 1920. Etude sur la prospection électrique du sous-sol. Gauthier-Villars. Tarasov, A., Titov, K., 2013. On the use of the Cole-Cole equations in spectral induced polarization. Geophys. J. Int. 195, 352-356.

Vallée, M.A., Smith, R.S., Keating, P., 2011. Metalliferous mining geophysics-state of the art after a decade in the new millennium. Geophysics 76, W31-W50.

Wang, T., Hohmann, G.W., 1993. A finite-difference, time-domain solution for three-dimensional electromagnetic modeling. Geophysics 58, 797-809.

Ward, S.H., Hohmann, G.W., 1988. Electromagnetic theory for geophysical applications. In: In: Nabighian, M.N. (Ed.), Electromagnetic Methods in Applied Geophysics: Theory, vol. 1. SEG, pp. 131-311.

Weidelt, P., 1982. Response characteristics of coincident loop transient electromagnetic systems. Geophysics 47, 1325-1330.

Zienkiewicz, O.C., Emson, C., Bettess, P., 1983. A novel boundary infinite element. Int. J. Numer. Methods Eng. 19, 393-404. 